OPEN ACCESS

Edited by: Uday Kishore,

Brunel University London, United Kingdom

Reviewed by:

Anuvinder Kaur,

Brunel University London, United Kingdom Taruna Madan,

National Institute for Research in Reproductive Health (ICMR), India

*Correspondence: Marko Salmi marko.salmi@utu.fi

†These authors share first authorship

Specialty section:

This article was submitted to

Molecular Innate Immunity,

a section of the journal

Frontiers in Immunology

Received: 18 June 2018 Accepted: 11 September 2018

Published: 08 October 2018

Citation:

Dunkel J, Viitala M, Karikoski M, Rantakari P, Virtakoivu R, Elima $K$, Hollmén M, Jalkanen S and Salmi M (2018) Enhanced Antibody Production in Clever-1/Stabilin-1-Deficient Mice.

Front. Immunol. 9:2257.

doi: 10.3389/fimmu.2018.02257

\section{Enhanced Antibody Production in Clever-1/Stabilin-1-Deficient Mice}

\author{
Johannes Dunkel ${ }^{1,2 t}$, Miro Viitala ${ }^{1,2 t}$, Marika Karikoski ${ }^{1,2}$, Pia Rantakari ${ }^{1,2}$, \\ Reetta Virtakoivu ${ }^{1,2}$, Kati Elima ${ }^{1,2}$, Maija Hollmén ${ }^{1,2}$, Sirpa Jalkanen ${ }^{1,2}$ and Marko Salmi ${ }^{1,2 *}$ \\ ${ }^{1}$ MediCity Research Laboratory, University of Turku, Turku, Finland , ${ }^{2}$ Institute of Biomedicine, University of Turku, Turku, \\ Finland
}

Clever-1, encoded by the Stab1 gene, is a scavenger and leukocyte trafficking receptor expressed by subsets of vascular and lymphatic endothelial cells and immunosuppressive macrophages. Monocyte Clever-1 also modulates T cell activation. However, nothing is known about the possible links between B cell function and Clever-1. Here, we found that Stab1 knockout mice (Stab1-/-) lacking the Clever-1 protein from all cells present with abnormally high antibody levels under resting conditions and show enhanced humoral immune responses after immunization with protein and carbohydrate antigens. Removal of the spleen does not abolish the augmented basal and post-immunization antibody levels in Clever-1-deficient mice. The increased IgG production is also present in mice in which Clever-1 is selectively ablated from macrophages. When compared to wildtype macrophages, Clever-1-deficient macrophages show increased TNF- $\alpha$ synthesis. In co-culture experiments, monocytes/macrophages deficient of Clever-1 support higher lgM production by B cells, which is blocked by TNF- $\alpha$ depletion. Collectively, our data show that the excessive inflammatory activity of monocytes/macrophages in the absence of Clever-1 results in augmented humoral immune responses in vivo.

Keywords: humoral immune responses, scavenging receptors, macrophages, B cells, immunoglobulins

\section{INTRODUCTION}

Clever-1 (common lymphatic endothelial and vascular endothelial receptor-1), a large $280 \mathrm{kDa}$ multidomain glycoprotein receptor encoded by the Stab1 gene, is associated with scavenging and leukocyte trafficking (1). Clever-1 (also known as stabilin-1 and Feel-1) is expressed by lymphatic vessels and non-continuous vascular endothelial cells in various organs such as the spleen, bone marrow, and liver, as well as by subsets of immunosuppressive macrophages (2-5). Clever-1 is a scavenger receptor with multiple ligands, including SPARC (secretory protein, acidic, and rich in cysteine), placental lactogen and oxidized, and acetylated low-density lipoprotein (6-10). The ability of Clever-1 to modulate angiogenesis and to bind bacteria in transfected cell lines has also been reported (4). Moreover, Clever-1 on lymphatic and vascular endothelia is involved in leukocyte-endothelium interactions (5, 11-14). In macrophages, Clever-1 supports their adhesion to the vascular endothelium under shear stress (15). In humans, Clever- $1^{\text {high }}$ monocytes display an anti-inflammatory gene signature and induce lower $\gamma$ interferon (IFN $\gamma$ ) and tumor necrosis factor- $\alpha$ (TNF- $\alpha)$ production in $\mathrm{T}$ cell antigen recall assays when compared to Clever- $1^{\text {low }}$ monocytes (16).

In contrast to macrophage and $\mathrm{T}$ cell biology, the potential functions of Clever-1 during humoral immune responses remain completely unknown. In addition to reactive antibodies against newly 
encountered antigens, humoral immunity also includes natural antibodies and antibodies from previous infections or vaccinations $(17,18)$. Natural $\mathrm{T}$ cell-independent (TI) antibodies are mainly produced by marginal zone B cells (MZB) in the spleen and B1 cells in the pleural and peritoneal cavities (19-21). Classical T cell-dependent (TD) antibody responses against protein antigens, in contrast, require concurrent activation of $\mathrm{CD}^{+}$helper $\mathrm{T}$ cells and $\mathrm{B}$ cells in lymphoid organs (22).

The aim of this work was to study the potential effects of Clever-1 on humoral immune responses. For that purpose, we evaluated natural and elicited TI and TD antibody responses, antigen scavenging and the collaboration of B cells with wildtype and Clever-1-deficient monocytes/macrophages in vivo and in vitro. We observed that the enhanced inflammatory activity of Clever-1-deficient monocytes/macrophages is associated with augmented natural and induced antibody production.

\section{MATERIALS AND METHODS}

\section{Mice}

All animal work was conducted in the Central Animal Laboratory, University of Turku, Turku, Finland. Clever-1 knockout $\left(\mathrm{Stab1}^{-/-}\right)$and macrophage-specific Lyz2Cre/Stab1 $1^{\mathrm{fl} / \mathrm{fl}}$ mice have been previously described (16). Age-matched wildtype, Stab1 ${ }^{-/-}$AND $L y z 2-C r e / S t a b 1^{\mathrm{fl} / \mathrm{fl}}$ mice were used in all experiments. All experiments were performed at least in duplicate, with a minimum of three mice per group, and the displayed results were pooled whenever possible. Mice were typically 6-8 weeks old at the beginning of experiments.

\section{Immunizations}

NP-Ficoll (conjugation ratio 4:9) and NP-KLH (conjugation ratio 2:3, both from Biosearch Technologies) were dissolved in sterile PBS (Sigma). $25 \mu \mathrm{g}$ of NP-Ficoll in $200 \mu \mathrm{l}$ PBS or $100 \mu \mathrm{g}$ of $\mathrm{NP}-\mathrm{KLH}$ in $200 \mu \mathrm{l}$ of PBS/alum adjuvant (mixed at a 1:1 ratio, from Thermo Fisher) was injected into mice intraperitoneally. Blood samples were collected into heparin-coated capillaries by tail vein punctures on indicated days. Plasma was separated by centrifugation and frozen. In certain experiments, the mice were sacrificed 7 days after immunization and tissues were collected for analyses.

\section{Analysis of Antibody Responses}

Total IgM and IgG levels were measured with commercial ELISA kits from Thermo Fisher, according to manufacturer's instructions.

(4-hydroxy-3-nitrophenyl)acetyl (NP)-specific IgG, IgM and IgG3 levels were measured with indirect ELISA, which was optimized in-house. Briefly, Nunc MaxiSorp plates were coated overnight with NP-BSA (conjugation ratio 4:1, from Biosearch Technologies) and blocked with 1\% BSA in PBS at RT for $2 \mathrm{~h}$. Samples were diluted in $1 \%$ BSA in PBS, added to the plates in duplicate and incubated at $+4^{\circ} \mathrm{C}$ overnight. Pooled plasma from immunized mice was used as standards for concentration curves. Polyclonal HRP-conjugated secondary antibodies (antimouse IgM, IgG, and IgG3, all from Southern Biotech) were diluted in $1 \%$ BSA in PBS, added to the plates and incubated at RT for $2 \mathrm{~h}$. NP-reactive antibodies were visualized by adding TMB chromogen (Invitrogen) and the reaction was stopped with $0.2 \mathrm{M}$ hydrochloric acid. Absorbance was measured with Tecan Infinite M200 and analyzed with the Magellan software (both from Tecan).

\section{Antibodies and Flow Cytometry}

Leukocytes from the spleen and bone marrow were isolated using mechanical teasing as described (23). Cells from the peritoneal cavity were obtained by peritoneal lavage. Blood leukocytes were analyzed from the EDTA-anticoagulated whole blood.

The following fluorochrome-conjugated monoclonal antimouse antibodies from BD were used: $\alpha$-CD4-APC-Cy7, $\alpha$-CD5FITC, $\alpha$-CD $8 \alpha$-PerCP-Cy5.5, $\alpha$-CD11b-PE, $\alpha$-CD19-FITC, $\alpha$ CD21/35-PE, $\alpha$-CD23-BV510, $\alpha$-CD24-BV650, $\alpha$-CD43-BV605, $\alpha$-CD44-PerCP-Cy5.5, $\alpha$-CD45-PE, $\alpha$-B220-BV421, $\alpha$-CD62LAlexa Fluor 647, $\alpha$-CD138-BV510, $\alpha$-Fas-PE, $\alpha$-fluoresceinAlexa Fluor 488, $\alpha$-IgM-APC-Cy7, $\alpha$-IgD-Alexa Fluor 647, and $\alpha$-Ly6C-BV421. Regulatory $\mathrm{T}$ cells were stained using the Mouse Regulatory $\mathrm{T}$ Cell Staining Kit 1 (eBioscience). The mStab1-1.26 (24) and isotype-matched control MOPC-21 (Bio X Cell) antibodies were conjugated with the Alexa Fluor 647 Antibody Labeling Kit according to the manufacturer's instructions (Thermo Fisher). Fc receptors were blocked with mouse Fc Block (BD) before staining with specific antibodies. Samples were run on LSRII or LSRFortessa (both from BD) and analyzed with the FlowJo software version 10 (Treestar).

The specific lymphocyte populations were gated as follows: MZB: CD $19^{+} \mathrm{CD} 21 / \mathrm{CD} 35^{\text {high }} \mathrm{CD} 23^{\text {low }}$. FoB: $\mathrm{CD} 19^{+}$ $\mathrm{CD} 21 / \mathrm{CD} 35^{\text {low }} \mathrm{CD} 23^{\text {high }}$. GCB: $\mathrm{B} 220^{+}$Fas ${ }^{\text {high }}$. Plasma cells: $\mathrm{CD} 5^{+}$B220 $20^{\text {low }} \mathrm{CD} 138^{\text {high }}$. B1 cells: $\mathrm{CD} 19^{+} \mathrm{CD}^{+/-} \mathrm{CD} 11 \mathrm{~b}^{+}$. Pre-pro B cells: $\mathrm{B} 220^{+} \mathrm{CD} 24^{\text {low }} \mathrm{CD} 43^{+}$. Pre-B cells: $\mathrm{B} 220^{+}$ $\mathrm{CD} 24^{+} \mathrm{CD}^{-} 3^{-} \mathrm{IgM}^{-} \mathrm{IgD}^{-}$. Immature B cells: B220 ${ }^{+} \mathrm{CD} 24^{+}$ $\mathrm{CD}^{-} 3^{-} \mathrm{IgM}^{\text {low }} \mathrm{IgD}^{-}$. Transitional B cells: $\mathrm{B} 220^{+} \mathrm{CD}^{+} 4^{+}$ $\mathrm{CD}^{2}{ }^{-} \mathrm{IgM}^{+} \mathrm{IgD}^{-}$. Early mature B cells: $\mathrm{B} 220^{+} \mathrm{CD}^{+} 4^{+}$ $\mathrm{CD}^{-}{ }^{-} \mathrm{IgM}^{+} \mathrm{IgD}^{+}$. Late mature B cells: $\mathrm{B} 220^{+} \mathrm{CD}_{2} 4^{+} \mathrm{CD}^{-} 3^{-}$ $\mathrm{IgM}^{\text {low }} \mathrm{IgD}^{+}$. Naïve $\mathrm{T}$ cells: $\mathrm{CD} 4^{+} / \mathrm{CD}^{+} \mathrm{CD} 44^{\text {low }} \mathrm{CD} 62 \mathrm{~L}^{\text {high }}$ Central memory $\mathrm{T}$ cells: $\mathrm{CD} 4^{+} / \mathrm{CD}^{+} \mathrm{CD} 44^{\text {high }} \mathrm{CD} 62 \mathrm{~L}^{\text {high }}$. Effector T cells: $\mathrm{CD} 4^{+} / \mathrm{CD} 8^{+} \mathrm{CD} 44^{\text {high }} \mathrm{CD} 62 \mathrm{~L}^{\text {low }}$. Regulatory T cells: $\mathrm{CD}^{+}{ }^{+} \mathrm{CD} 25^{+} \mathrm{FoxP}^{+}$. Isotype-matched negative control antibodies conjugated to the same fluorochrome were used to determine gating.

\section{Anti-Clever-1 Uptake}

Blood was collected by cardiac puncture and the red blood cells removed with Pharm Lyse lysing solution (BD). Leukocytes were incubated at $37^{\circ} \mathrm{C}$ for $1 \mathrm{~h}$ with $20 \mu \mathrm{g} / \mathrm{ml}$ of either mStab1-1.26or MOPC-21-Alexa Fluor 647 (conjugated as described above), washed, stained with $\alpha$-CD11b-PE and $\alpha$-Ly6C-BV421 and analyzed by flow cytometry. The geometric mean fluorescence intensities of the Ly6C $\mathrm{C}^{\text {high }}$ and Ly6 $\mathrm{C}^{\text {low }}$ monocyte populations of wildtype mice were normalized to the unspecific antibody binding of the corresponding populations of Stab1 ${ }^{-/-}$mice, which were set at $100 \%$. 


\section{Microscopy}

Eight micrometer cryosections were cut and fixed in $-20^{\circ} \mathrm{C}$ acetone for $5 \mathrm{~min}$. Primary unconjugated or biotin-conjugated antibodies against CD169, CD209b, F4/80, Meca32 (anti-PV-1) and 9-11 (anti-Clever-1) (11) were diluted in 2\% plasma in PBS. Species-specific polyclonal secondary antibodies or streptavidin conjugated with Alexa Fluor 488 or Alexa Fluor 546 (Life Technologies) diluted 1:1,000 in PBS were used for detection as appropriate. In certain experiments, Alexa Fluor 647-conjugated Meca32 was used. Imaging was performed with an Olympus BX60 wide-field microscope or a Zeiss LSM780 confocal microscope equipped with a motorized stage. Images were analyzed with the Image J software version $1.50 \mathrm{~b}(\mathrm{NIH})$ or the Imaris software version 8 (Bitplane). Fluorescence values were thresholded and the background was subtracted with reference to isotype control staining.

Hematoxylin/eosin-stained spleen paraffin sections were scanned with a Panoramic 250 Slide Scanner and analyzed with the CaseViewer software (both from 3DHISTEC).

Cells were cultured in vitro on coverslips and fixed with $4 \%$ paraformaldehyde and permeabilized and blocked with $0.1 \%$ Triton X in PBS with $30 \%$ horse serum for $20 \mathrm{~min}$. After three washes with PBS, $\alpha$-TNF- $\alpha$-Alexa Fluor 488 (Thermo Fisher), and mStab1.26-Alexa Fluor 647 (conjugated as described above) antibodies diluted 1:200 were added for $1 \mathrm{~h}$. After washes, nuclei were stained with Hoechst. Coverslips were mounted with Vectashield mounting medium. Confocal 3D images were captured with Zeiss Axiovert $200 \mathrm{M}$ with the spinning disk confocal unit Yokogawa CSU22 and the Zeiss Plan-Neofluar 63 $\times$ oil/1.4 NA objective. Images were analyzed with the ImageJ software.

\section{Splenectomy}

Mice were anesthetized with intraperitoneal ketamine (Orion Pharma) and xylazine (Bayer). Buprenorphine (Orion Pharma) was injected subcutaneously for perioperative analgesia. The peritoneal cavity was opened at the left flank and the spleen was removed by thermo-cauterization. The peritoneum and skin were closed with absorbable suture material. Carprophen was injected subcutaneously once daily for postoperative analgesia.

\section{Leukocyte Enumeration}

Blood was drawn by tail vein puncture 30 days after splenectomy or sham surgery and peripheral blood cell populations were quantified with a VetScan hematology system (Abaxis).

\section{In vivo Antigen Capture}

NP-AECM-Ficoll-fluorescein (conjugation ratio 94:11, from Biosearch Technologies) was diluted in PBS and injected intravenously. Mice were sacrificed $45 \mathrm{~min}$ or 7 days after the injections and the spleens were collected and frozen in OCT (Sakura) for sectioning.

\section{M2 Macrophage Culture}

Bone marrow cells from adult wildtype and Stab1 $1^{-/-}$mice were cultured in IMDM supplemented with 10\% FCS, $1 \%$ penicillin/streptomycin and $20 \mathrm{ng} / \mathrm{ml} \mathrm{M-CSF}$ (PeproTech). On day 4, dexamethasone was added to $100 \mathrm{nM}$ and the cells cultured for an additional 3 days. To induce TNF- $\alpha$ production, LPS was added to $50 \mathrm{ng} / \mathrm{ml}$ for $5 \mathrm{~h}$. The cells were treated with $10 \mu \mathrm{g} / \mathrm{ml}$ of brefeldin A to inhibit cytokine secretion.

\section{Co-culture Experiments}

Splenocytes and bone marrow cells were collected as described above and passed through Lympholyte-M density gradients to remove dead cells (Cedarlane). Splenic B cells were isolated with the B Cell Isolation Kit (mouse), from which CD $138^{+}$ plasma cells were depleted with CD138 Microbeads (mouse) according to the manufacturer's instructions. Monocytes were isolated with the Monocyte Isolation Kit (Bone Marrow) (mouse) according to the manufacturer's instructions (all kits were from Miltenyi Biotec). Purity of the isolated cells was assessed by flow cytometry. Isolated B cells were $90 \% \mathrm{CD} 45 \mathrm{R}^{+} \mathrm{CD} 138^{-}$and isolated monocytes $90 \% \mathrm{CD} 11 \mathrm{~b}^{+} \mathrm{Ly}_{6 \mathrm{C}} \mathrm{Cigh}^{\text {high }}$ Isolated cells were plated at $0.5 \times 10^{6}$ cells/ml in RPMI-1640 supplemented with $10 \%$ FCS, $1 \%$ penicillin/streptomycin and $1 \%$ Glutamax in the indicated combinations and incubated for 5 days, after which the supernatants were collected for ELISA (described above). The neutralizing $\alpha$-TNF- $\alpha$ antibody clone XT3.11 (Bio X Cell) was used at $50 \mu \mathrm{g} / \mathrm{ml}$.

\section{RNA Sequencing and Quantitative PCR}

Total RNA from dissected livers of E17.5 and 2-weeks-old wildtype ( $n=4$ for both time points) and $\operatorname{Stab1}^{-/-}(n=3$ for E17.5 and 4 for 2 weeks) mice was isolated and RNA-seq performed as described in (25). The RNA-seq results have been deposited into the Gene Expression Omnibus database (accession number GSE114969). For qPCR, total RNA was isolated from adult wildtype and Stab1 $1^{-/-}$mouse spleens $(n=3)$ using the RNeasy Kit (Qiagen) and reverse transcribed to cDNA with the SuperScript VILO cDNA Synthesis Kit (ThermoFisher Scientific) according to the manufacturers' instructions. Quantitative PCR was carried out using Taqman Gene Expression Assays (Thermo Fisher) for Ifi202b (Mm00839397_m1; target gene), and Actb (Mm00607939_s1; control gene). The reactions were run using a QuantStudio12K Flex Real-Time PCR system (Thermo Fisher) at the Finnish Microarray and Sequencing Centre, Turku Centre for Biotechnology. Relative quantification was performed using the $2^{-\Delta \Delta C T}$ method, in which the gene expression level in knockout mice is compared to that in wildtype mice, in which $\mathrm{RQ}=1$.

\section{Statistical Analyses}

Experimental data are presented as scatter plots with bars representing the means \pm standard errors of the means (s.e.m.), excepting kinetic experiments for NP-specific antibody titers, which are presented as means \pm s.e.m. Probabilities were calculated with Student's unpaired two-tailed $t$-test with Welch's correction when applicable or with two-way ANOVA followed by Bonferroni's multiple comparisons tests using GraphPad Prism 7 (GraphPad Software). Threshold of statistical significance was set at $P<0.05$. 


\section{RESULTS}

\section{Elevated Plasma IgM and IgG Levels in the Absence of Clever-1}

The role of Clever-1 in B cell biology has not been previously studied. Therefore, we initially measured the baseline plasma concentrations of IgM and IgG in wildtype and Stab1-/- mice that lack Clever- 1 from all cells. ELISA revealed that the baseline levels of total IgM were increased by $38.7 \pm 5.4 \%$ and total IgG by $67.2 \pm 14.0 \%$ in Stab1 $1^{-/}$mice compared to wildtype mice $(P=0.0327$ and 0.0425 , respectively) (Figures 1A,B). Thus, the genetic deletion of Clever-1 resulted in constitutively increased basal levels of both IgM and IgG in vivo.

\section{Accelerated TD and TI-2 Antibody Responses in Stab1-/- Mice}

To study the role of Clever- 1 in classical TD antibody production, we used intraperitoneal nitrophenyl (NP)-KLH immunization with adjuvant (Figure 1C). Kinetic measurements of plasma antibody levels showed that the NP-KLH-specific IgM response was accelerated in $S t a b 1^{-/-}$mice compared to wildtype mice (Figure 1D). On day 7, the level of NP-specific IgM was 0.46 \pm 0.034 relative units (RU) in wildtype mice and $0.69 \pm 0.048$ RU in Stab1 $1^{-/}$mice $(P=0.0742)$, and on day 14 , the NPspecific IgM levels reached $0.56 \pm 0.062 \mathrm{RU}$ and $0.81 \pm 0.084$ RU in wildtype and Stab1 $1^{-/-}$mice, respectively $(P=0.0496)$ (Figure 1D). The level of NP-specific IgG was $52.8 \pm 16.3 \%$ higher on day 21 after NP-KLH immunization in Stab1-/mice compared to wildtype mice, although the differences were not statistically significant at any measured time point (Figure 1E).

TI antibody responses were studied with the TI-2 antigen NP-Ficoll (Figure 1F). We observed that the NP-specific IgM response was more robust in Stab1 $1^{-/}$mice compared to wildtype mice (Figure 1G). On day 9, the level of NP-specific IgM was $1.16 \pm 0.10 \mathrm{RU}$ in wildtype mice and $2.09 \pm 0.22$ RU in Stab1 ${ }^{-/-}$mice $(P<0.0001)$, and on day 15 , the NPspecific IgM levels were $1.29 \pm 0.15 \mathrm{RU}$ in wildtype mice and $1.84 \pm 0.22 \mathrm{RU}$ in Stab1 $1^{-/-}$mice $(P=0.0335)$ (Figure 1G). Similarly, class switching to the NP-specific $\mathrm{IgG}_{3}$ isotype, which is relatively specific to TI-2 antibody responses (26), was enhanced in Stab1 $1^{-/-}$mice (Figure 1H). On day 15, the levels of NPspecific $\mathrm{IgG}_{3}$ remained at $0.50 \pm 0.070 \mathrm{RU}$ in wildtype mice but rose to $1.26 \pm 0.16 \mathrm{RU}$ in $\operatorname{Stab} 1^{-/-}$mice $(P<0.0001)$ (Figure 1H). Collectively, these data show that both TD and especially TI-2 antibody responses are enhanced in mice lacking Clever-1.

\section{Altered Splenic B Cell Subpopulations in Stab1-/- Mice}

Since the spleen plays a central role in humoral immunity, we next compared splenic B cell populations between wildtype and Stab1-/- mice. The frequencies of $\mathrm{B} 220^{+}$ B cells did not differ significantly between wildtype and Stab1 ${ }^{-/-}$mice, nor did their absolute numbers per $\mathrm{mg}$ of spleen (Figures 2A,B). Nevertheless, the spleens of Stab1-/-

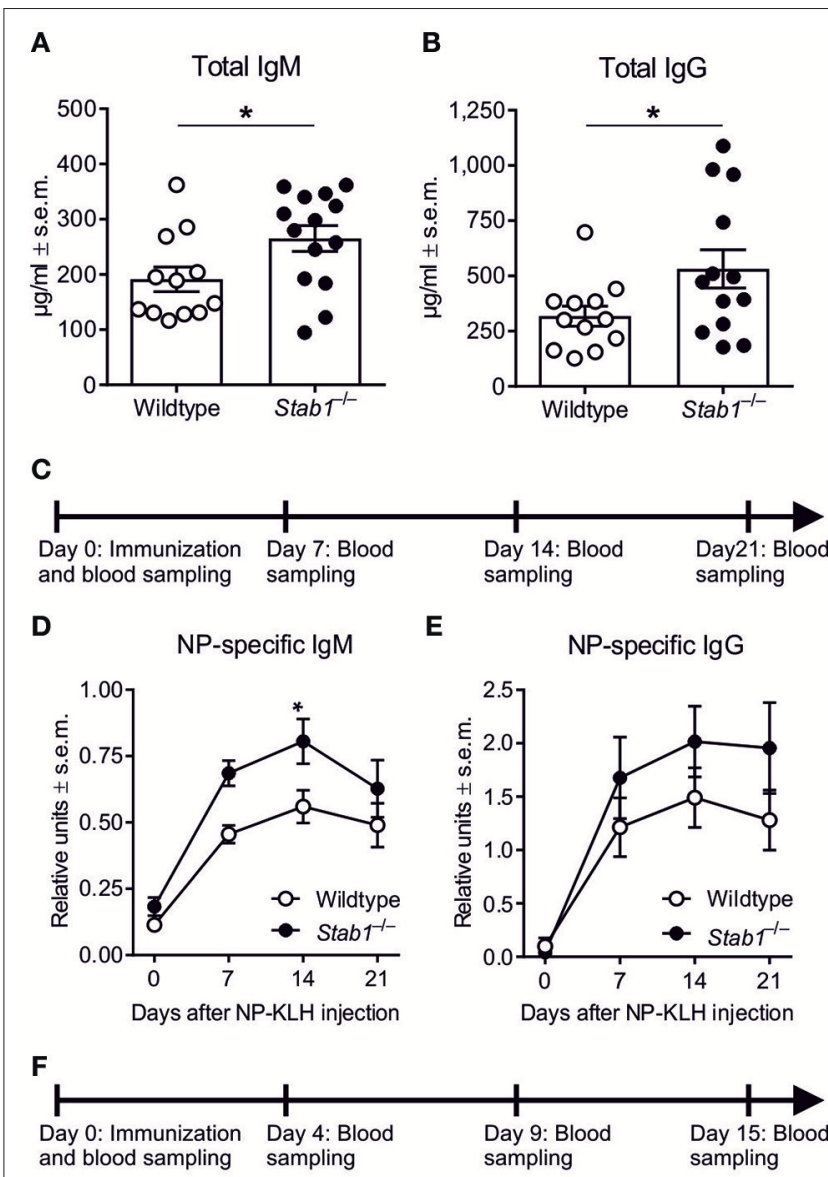

G

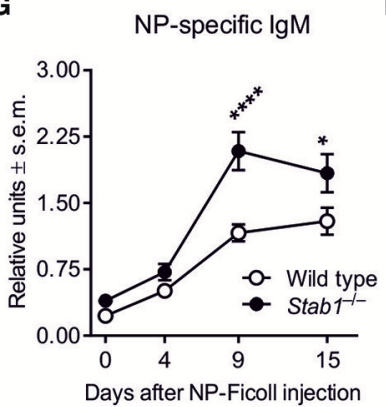

H

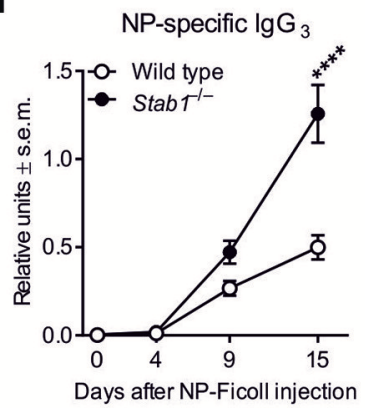

FIGURE 1 | Stab1-/- mice have elevated antibody levels at baseline and after immunization with TD and TI antigens. (A,B) Baseline plasma concentrations of IgM (A) and IgG (B) as determined by ELISA. (C) Experimental protocol for NP-KLH (TD antigen) immunization. (D,E) Kinetics of NP-specific IgM (D), and IgG (E) production after NP-KLH immunization (RU, relative units) determined by ELISA. (F) Experimental protocol for NP-Ficoll (TI-2 antigen) immunization. (G-H) Kinetics of NP-specific IgM (G) and Ig $\mathbf{G}_{3}$ (H) production after NP-Ficoll immunization in wildtype and Stab1-/- mice. $(\mathbf{A}, \mathbf{B})$ Each data point represents one mouse and bars represent the means \pm s.e.m., $n=12$ (wildtype) and 14 (Stab1-/-) (A) and 12 (wildtype) and 13 $\left(S t a b 1^{-/-}\right)$(B). Statistical significance was determined with Student's unpaired two-tailed $t$-test (A) and Student's unpaired two-tailed $t$-test with Welch's correction (B), ${ }^{*} P<0.05$. (D,E, G,H) Data are presented as means \pm s.e.m., $n=10-11$ (wildtype) and 8-9 (Stab1-/-) (D, E), 12 (wildtype) and 15 $\left(\right.$ Stab $\left.1^{-/-}\right) \mathbf{( G )}$ and 4 (wildtype) and $5\left(\right.$ Stab $\left.1^{-/-}\right) \mathbf{( H )}$. Statistical significance was determined with two-way ANOVA followed by Bonferroni's multiple comparisons tests, ${ }^{\star} P<0.05,{ }^{\star \star \star \star} P<0.0001$. 


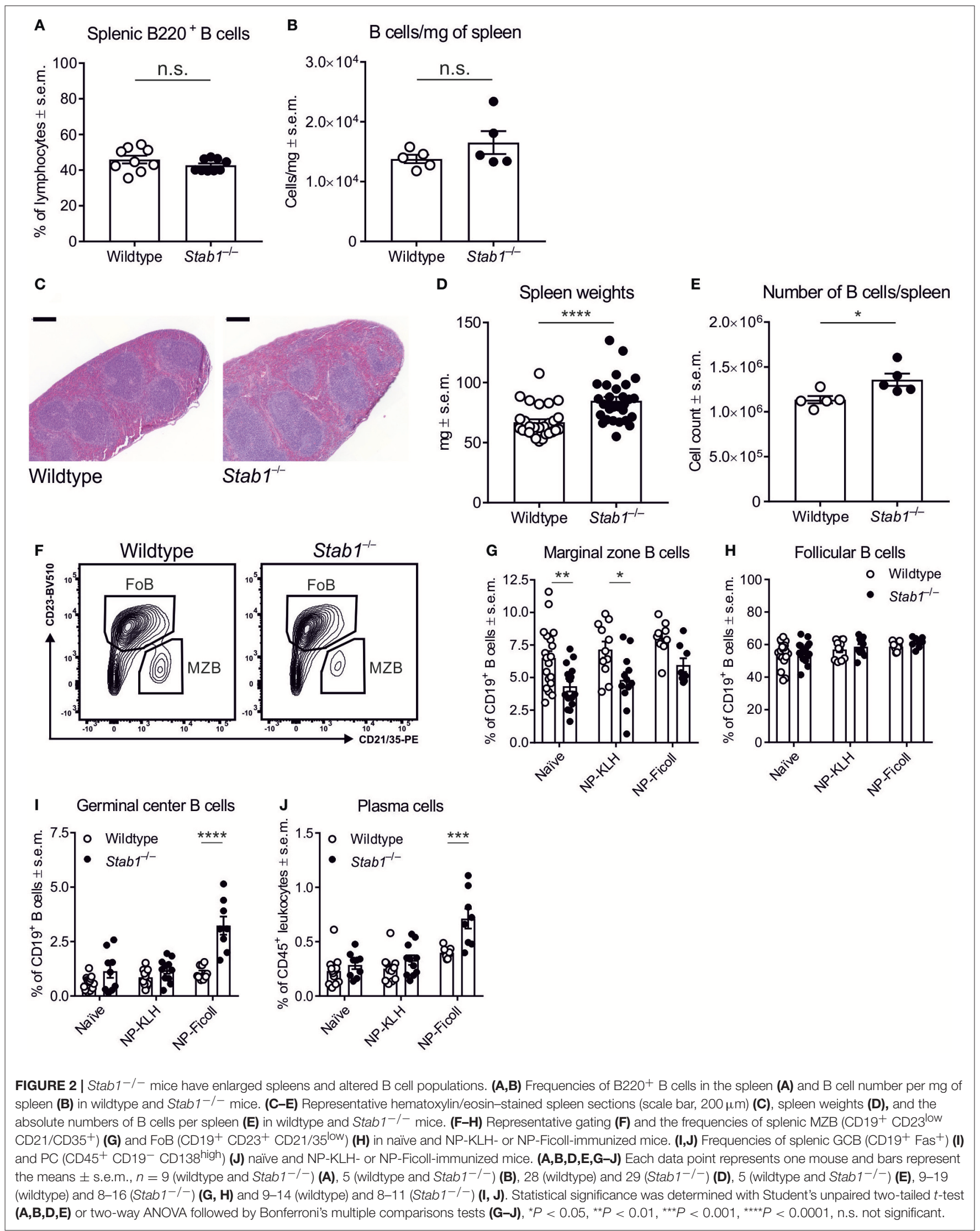


mice were on average larger than those of wildtype mice $(P<0.0001)$, increasing the absolute number of $\mathrm{B}$ cells per spleen (Figures 2C-E). Unexpectedly, in spite of the increased natural $\mathrm{TI}$ antibody levels in $S t a b 1^{-/-}$mice, the frequency of MZB was reduced by $33.3 \pm 4.0 \%$ in naïve Stab1 $1^{-/}$mice compared to naïve wildtype mice $(P=0.0041)$ (Figures 2F,G). The frequencies of splenic follicular B cells (FoB) and $\mathrm{CD}^{+}{ }^{+}$and $\mathrm{CD} 8^{+} \mathrm{T}$ cells were comparable between naive wildtype and $S t a b 1^{-/-}$mice, although the frequency of naïve $\mathrm{CD}^{+} \mathrm{T}$ cells in the spleen was increased in Stab1-/mice compared to wildtype mice $(P<0.0001)$ (Figure $2 \mathrm{H}$ and Supplementary Figures S1A-C).

After immunization with NP-KLH, MZB remained scarcer by $32.5 \pm 4.7 \%$ in $S t a b 1^{-/-}$mice compared to wildtype mice $(P=0.0147)$, but we did not observe statistically significant differences in the frequencies of FoB in response to either TD or TI antigen (Figure 2G and $\mathbf{H}$ ). In contrast, after immunization with NP-Ficoll, the frequencies of reactive germinal center B cells (GCB) and plasma cells (PC) were robustly elevated by $203.4 \pm 31.1 \%$ and $79.0 \pm 9.9 \%$, respectively, in Stab1 $1^{-/}$ mice compared to wildtype mice $(P<0.0001$ and $P=0.0001$, respectively) (Figures $2 \mathbf{I}, \mathbf{J})$. Thus, Stab1 ${ }^{-/-}$mice manifest with constitutive hyperimmunoglobulinemia in the absence of increased frequencies of MZB or FoB in the spleen.

\section{Extrasplenic Factors Contribute to Hyperimmunoglobulinemia in Stab1-/- Mice}

Clever-1 is generally expressed by subsets of endothelial cells and immunosuppressive macrophages (2-5). In the spleen, however, we found that Clever-1 expression was strictly limited to PV-1 ${ }^{+}$ vascular endothelial cells in the red pulp (Figure 3A). No antiClever-1 staining of spleen sections co-localized with CD209b ${ }^{+}$ marginal zone (MZ) macrophages, $\mathrm{CD}_{169^{+}}$metallophilic macrophages or $\mathrm{F} 4 / 80^{+}$red pulp macrophages (Figure 3A). The specificity of the anti-Clever-1 antibody was confirmed by the lack of reactivity with any cell type in spleens from $S t a b 1^{-/-}$mice (Supplementary Figure S2).

We then tested whether the accelerated antibody responses in Stab1 $1^{-/}$mice might be attributed to better antigen access to the marginal zone due to systemically absent Clever-1dependent scavenging. However, we saw no differences in the accumulation of intravenously injected NP-Ficoll into CD209b ${ }^{+}$ MZ macrophages between wildtype and Stab1-/- mice after $45 \mathrm{~min}$, reflecting immediate antigen access, or after 7 days, reflecting long-term antigen accumulation (Figures 3B,C). Thus, in the spleen, Clever-1 is exclusively expressed by vascular endothelial cells, and Clever-1 deficiency does not affect the delivery of intravascular TI antigen to the marginal zone.

To further analyze the possible contribution of the spleen to the altered humoral immune responses observed in $S t a b 1^{-/-}$mice, we studied antibody production in response to immunization after splenectomy (Figure 3D). As expected, splenectomy lowered basal IgM levels in both wildtype and Stab1 $1^{-/}$mice when compared to sham-operated mice by 65.8 $\pm 14.9 \%$ and $70.1 \pm 18.2 \%$, respectively, whereas basal IgG levels remained unaffected (Figures 3E,F). Notably, even after splenectomy, the basal levels of IgM and IgG remained 123.5 $\pm 26.1 \%$ and $191.4 \pm 39.0 \%$ higher, respectively, in Stab1 ${ }^{-/-}$ mice compared to wildtype mice $(P=0.0059$ and 0.0029 , respectively) (Figures 3E,F). Additionally, after immunization with NP-Ficoll, splenectomized Stab1 $1^{-/}$mice still produced NP-specific IgM more robustly than splenectomized wildtype mice, although these differences did not reach statistical significance (Figure 3G). Immunizing splenectomized mice did not further increase IgG levels in either genotype, but the IgG levels remained significantly higher in $S t a b 1^{-/-}$mice throughout the time course (Figure 3H). Our experiments on splenectomized mice therefore strongly suggest that the increased basal IgM and IgG and TI antigen-induced IgM levels in $S t a b 1^{-/-}$mice in comparison to wildtype mice are due to the effects of Clever-1 on B cell function mainly outside the spleen.

\section{Delayed B Lymphopoiesis, B Lymphocytemia and Diminished Peritoneal B1 Cells in Stab1-/- Mice}

In search of the extrasplenic origin of hyperimmunoglobulinemia in Stab1 $1^{-/-}$mice, we next analyzed B cell maturation in the bone marrow. Flow cytometric analysis revealed that the frequencies of pre-pro, pre and early mature B cells were reduced by 32.0 $\pm 3.4 \%, 35.9 \pm 6.8 \%$ and $24.5 \pm 0.7 \%$, respectively, in Stab1 $1^{-/-}$ mice compared to wildtype mice $(P=0.0140,0.0002$ and 0.0004 , respectively), whereas those of late mature and transitional $B$ cells were comparable between the two genotypes (Table 1). The frequencies of PC and T cell subpopulations in the bone marrow were comparable between wildtype and Stab1 ${ }^{-/-}$mice (Table 1 and Supplementary Table 1).

In the blood, the frequency of $\mathrm{B}_{2} 20^{+} \mathrm{B}$ cells among lymphocytes was $43.5 \pm 6.0 \%$ higher in Stab1 $1^{-/}$mice compared to wildtype mice $(P=0.0147)$ (Table 1$)$, whereas the frequency of $\mathrm{CD}^{+} \mathrm{T}$ cells was $38.8 \pm 7.0 \%$ lower, $(P=0.0227)$ (Supplementary Table 1). Otherwise, the numbers of total leukocytes, lymphocytes and granulocytes in the blood of wildtype and Stab1 $1^{-/-}$mice $\left(6.4 \times 10^{3}, 5.7 \times 10^{3}\right.$, and $0.67 \times$ $10^{3}$ cells $/ \mu l$ in wildtype mice vs. $7.0 \times 10^{3}, 6.1 \times 10^{3}$, and 0.75 $\times 10^{3}$ cells $/ \mu \mathrm{l}$ in $S t a b 1^{-/-}$mice, respectively) were comparable between the two genotypes, as were the frequencies of total blood $\mathrm{CD}^{+} \mathrm{T}$ cells and blood $\mathrm{CD} 4^{+}$and $\mathrm{CD} 8^{+} \mathrm{T}$ cell subpopulations (Supplementary Table 1).

Because B1 cells also contribute to antibody production, we studied their abundance in the bone marrow and peritoneum in Stab1 $1^{-/-}$mice. This analysis revealed comparable frequencies of bone marrow B1 cells between wildtype and Stab1-/mice, but a $43.0 \pm 6.2 \%$ decrease in the frequency of peritoneal B1 cells in Stab1 $1^{-/-}$mice compared to wildtype mice $(P=0.0032)$ (Table 1). Collectively, these data show alterations in early $\mathrm{B}$ cell lymphopoiesis in the bone marrow, in the numbers of circulating B cells and in peritoneal B1 cells in Stab1 ${ }^{-/-}$mice, yet these extrasplenic B cell aberrations do not apparently correlate with the hyperimmunoglobulinemia of these mice. 

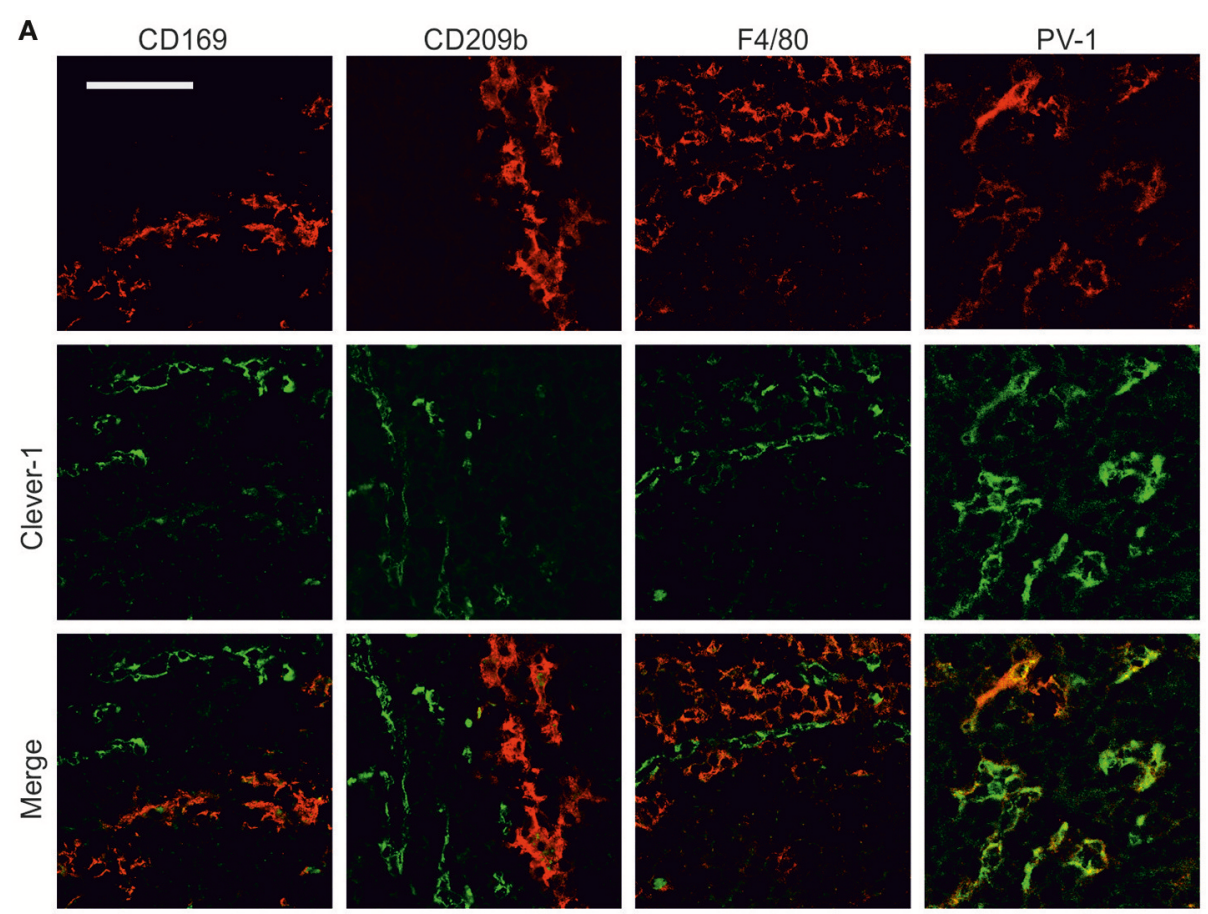

B

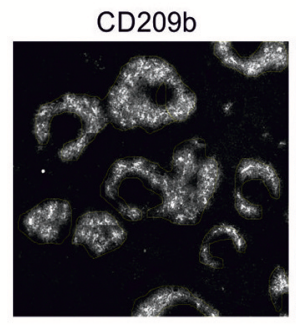

NP-Ficoll-fluorescein
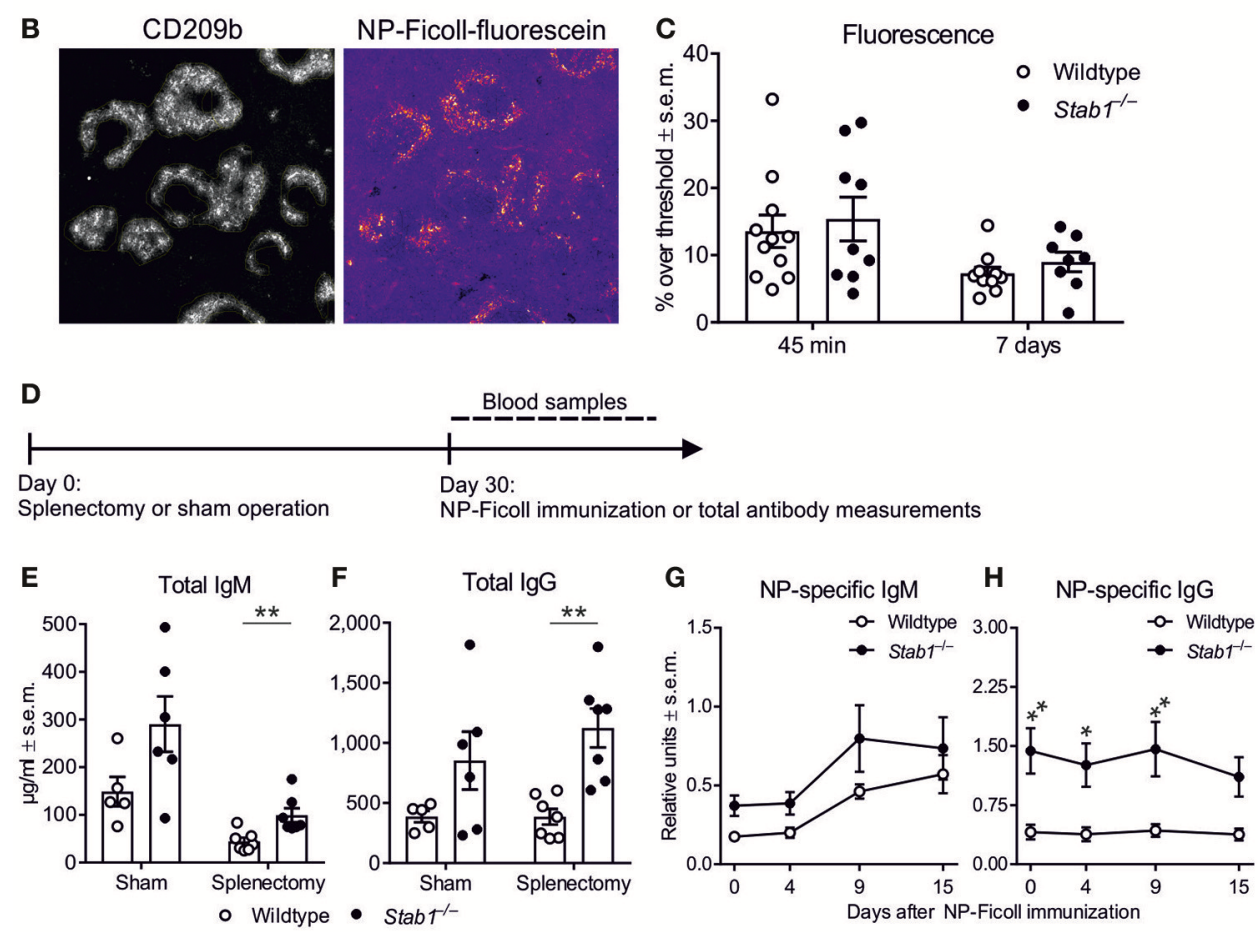

FIGURE 3 | The elevated antibody levels in Stab1-/- mice are regulated by extrasplenic factors. (A) Immunostaining of wildtype spleen sections with antibodies against Clever-1 (green, middle row) and CD169 (metallophilic macrophage marker), CD209b (MZ macrophage marker), F4/80 (red pulp macrophage marker) or PV-1 (vascular endothelial cell marker) (red, top row). Yellow color in the merged images indicates co-localization (bottom row) (scale bar, $200 \mu \mathrm{m})$. Images are representative of three independent experiments. (B,C) Representative immunostaining of fluorescein-labeled NP-Ficoll accumulation into CD209b+ $\mathbf{M Z}$ macrophages (B) and quantification at $45 \mathrm{~min}$ and 7 days (C) after intravenous injection. (D) Experimental protocol for splenectomy and NP-Ficoll immunization. $(\mathbf{E}, \mathbf{F})$ Plasma concentrations of $\lg M(\mathbf{E})$ and $\lg \mathbf{G}(\mathbf{F})$ after splenectomy or sham operation as determined by ELISA. (G,H) Kinetics of NP-specific lgM (G) and IgG (H) production after NP-Ficoll immunization in splenectomized mice. (C,E,F) Each data point represents one mouse and bars represent the means \pm s.e.m., $n=10-11$ (wildtype) and 8-9 (Stab1-/-) (C) and 5-7 (wildtype) and 6-7 (Stab1-/-) (E, F). (G, H) Data are presented as means \pm s.e.m., $n=4$ (wildtype and Stab1-/-). $\mathbf{( C , E - H ) ~ S t a t i s t i c a l ~ s i g n i f i c a n c e ~ w a s ~ d e t e r m i n e d ~ w i t h ~ S t u d e n t ' s ~ u n p a i r e d ~ t w o - t a i l e d ~} t$-test with Welch's correction when applicable (C,E,F) or two-way ANOVA followed by Bonferroni's multiple comparisons tests $(\mathbf{G}, \mathbf{H}),{ }^{\star} P<0.05,{ }^{\star \star} P<0.01$. 
TABLE 1 | Phenotype of B cells in wildtype and Stab1-/- mice.

\begin{tabular}{|c|c|c|c|}
\hline & Wildtype & Stab1-/- & \\
\hline \multicolumn{4}{|l|}{ BONE MARROW } \\
\hline Pre-pro (B220+ CD24low $\mathrm{CD}^{+} 3^{+}$) & $15.8 \pm 1.1^{\mathrm{a}}(n=5)$ & $10.7 \pm 0.9^{a}(n=5)$ & $\star b$ \\
\hline Immature (B220+ CD24+ CD43- IgMlow $\left.\operatorname{lgD}^{-}\right)$ & $7.7 \pm 0.2^{\mathrm{a}}(n=5)$ & $7.7 \pm 0.2^{\mathrm{a}}(n=5)$ & \\
\hline Early mature $\left(\mathrm{B} 22 \mathrm{O}^{+} \mathrm{CD} 4^{+} \mathrm{CD} 3^{-} \operatorname{lgM}^{+} \operatorname{lgD}{ }^{+}\right)$ & $27.9 \pm 0.6^{\mathrm{a}}(n=5)$ & $21.1 \pm 0.4^{a}(n=5)$ & $\star \star \star b$ \\
\hline Plasma cells (CD45+ B220low CD138high) & $44.9 \pm 2.5^{\mathrm{a}}(n=5)$ & $44.1 \pm 0.8^{a}(n=5)$ & \\
\hline B1 cells $\left(\mathrm{CD} 19^{+} \mathrm{CD}^{+/-} \mathrm{CD} 11 \mathrm{~b}^{+}\right)$ & $3.2 \pm 0.5^{\mathrm{a}}(n=8)$ & $3.4 \pm 0.2^{\mathrm{a}}(n=9)$ & \\
\hline \multicolumn{4}{|l|}{ BLOOD } \\
\hline $\mathrm{B} 220^{+} \mathrm{B}$ cells & $42.2 \pm 5.3^{\mathrm{a}}(n=9)$ & $60.6 \pm 3.4^{\mathrm{a}}(n=9)$ & *C \\
\hline \multicolumn{4}{|l|}{ PERITONEAL CAVITY } \\
\hline
\end{tabular}

\section{Monocyte/Macrophage Clever-1 Regulates Antibody Production}

To analyze the potential role of macrophage Clever- 1 in the regulation of antibody production, we utilized our Lyz2-Cre/Stab $1^{\mathrm{fl} / \mathrm{fl}}$ mice that lack Clever-1 selectively from monocytes/macrophages (27). Notably, we observed increased plasma levels of IgG also in Lyz2-Cre/Stab $1^{\mathrm{f} / \mathrm{fl}}$ mice compared to wildtype mice (Figures 4A,B).

We have previously reported that in human monocytes/macrophages, silencing Stab1 with siRNA and blocking Clever-1 with a specific antibody associate with the increased production of inflammatory cytokines, including TNF- $\alpha(15,16)$. Incubation of peripheral blood leukocytes from wildtype and Stab1-/- mice with directly-conjugated antiClever-1 antibody mStab1-1.26 or an isotype-matched control antibody revealed specific Clever-1 expression by Ly6C $\mathrm{C}^{\text {high }}$ classical monocytes, but not by Ly6 $\mathrm{C}^{\text {low }}$ non-classical monocytes $(P=0.0482)$ (Figure 4C). Moreover, bone-marrow-derived monocytes/macrophages from Stab1 $1^{-/-}$mice produced higher levels of TNF- $\alpha$ than those from wildtype mice when stimulated with LPS $(P<0.0001)$ (Figures 4D,E). Thus, murine monocytes and monocyte-derived macrophages express Clever-1, and Clever-1 is functionally involved in the regulation of TNF- $\alpha$ synthesis.

To determine whether the heightened inflammatory propensity of Clever-1-deficient monocytes/macrophages could be linked to the increased IgM production observed in Stab1 $1^{-/-}$ mice, we performed co-culture experiments with wildtype splenic B cells (depleted of plasma cells) and wildtype or Stab1 $1^{-/-}$monocytes/macrophages isolated from bone marrow. Consistent with the fact that monocytes/macrophages regulate $B$ cell function through the secretion of cytokines such as TNF- $\alpha(28,29)$, ELISA of the co-culture supernatants showed increased secretion of IgM when either wildtype or Stab1 $1^{-/-}$ monocytes were co-cultured with B cells compared to B cells alone ( $P=0.0091$ and 0.0002 , respectively) (Figure 4F). Notably,
Stab1 ${ }^{-/-}$monocytes were able to induce $47.1 \pm 8.9 \%$ greater secretion of IgM by B cells compared to wildtype monocytes $(P=0.0171)$ (Figure 4F). The same effect was observed in another independent co-culture experiment $(P=0.0030)$. The increased IgM production was neutralized by TNF- $\alpha$ depletion in co-cultures with wildtype monocytes but only partially in co-cultures with Stab1 ${ }^{-/-}$monocytes, where the amount of secreted IgM remained significantly higher even after TNF- $\alpha$ depletion $(P=0.0321)$ (Figure 4F). Therefore, the contribution of other inflammatory factors whose production is increased in the absence of Clever-1 is likely. This notion is supported by the finding that interferon-activated gene 202b (Ifi202b) was upregulated in $S t a b 1^{-/-}$mice. RNA sequencing of liver samples from fetal and 2-weeks-old wildtype and Stab1 ${ }^{-/-}$mice showed that Ifi202b was not expressed in wildtype samples, but clearly expressed in samples from Stab1 $1^{-/-}$mice $\left(\log _{2}\right.$ Stab1 $1^{-/}$/wildtype $=8.64$ in fetal and 9.02 in 2-weeks-old mice) (Supplementary Table 2). Similarly, when assayed by $\mathrm{qPCR}$ from adult spleens, Ifi202b expression was negligible in wildtype mice but strongly induced in Stab1 ${ }^{-/-}$mice (RQ $\left.=267, n=3\right)$. Collectively, these experiments suggest that without Clever-1, monocytes/macrophages have an intrinsically heightened ability to stimulate B cells to produce antibodies.

\section{DISCUSSION}

We found here that Stab1-/- mice manifest with elevated basal levels of IgM and IgG and augmented TD and TI antibody responses. While the frequency of $\mathrm{B}$ cells in the blood of Stab1-/- mice was higher than in wildtype mice, the frequencies of $\mathrm{B}_{22} 20^{+} \mathrm{B}$ cells and $\mathrm{FoB}$ in the spleen were similar between the two genotypes, and the augmented antibody synthesis in the absence of Clever-1 was also observed in splenectomized mice. Therefore, Clever-1 does not seem to affect antibody production by controlling B cell numbers and humoral immune responses in the spleen. Instead, our functional data 

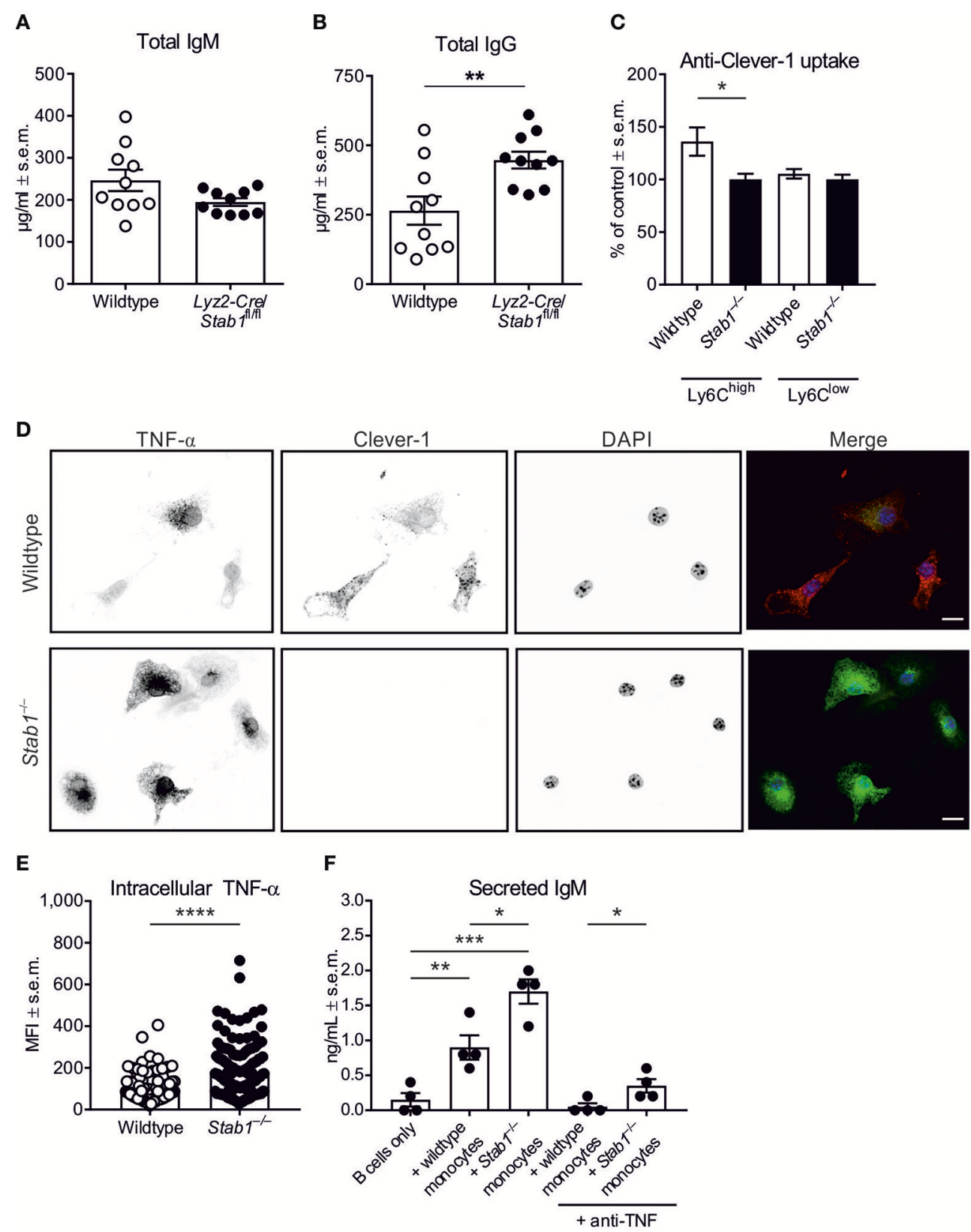

FIGURE 4 | Stab1-/- monocytes induce enhanced antibody production by B cells. (A,B) Baseline plasma concentrations of IgM (A) and IgG (B) in

Lyz2-Cre/Stab $1^{\mathrm{fl} / \mathrm{fl}}$ mice and control mice. (C) Uptake of fluorescently-conjugated anti-Clever-1 antibody by Ly6Chigh and Ly6Clow monocytes from naïve mice. MFI values have been normalized to unspecific antibody uptake by the corresponding Stab $1^{-/-}$monocyte populations, which have been set at $100 \%$. (D) Representative immunofluorescence images of wildtype and Stab1-/- M2 macrophages treated with LPS and brefeldin A for $5 \mathrm{~h}$. In the merged images, nuclei are shown in blue, TNF- $\alpha$ in green and Clever-1 in red (scale bar, $10 \mu \mathrm{m}$ ). (E) Quantification of TNF- $\alpha$ mean fluorescence intensities (MFI) per cell from (D). The quantification is representative of three independent experiments. (F) Concentrations of secreted IgM as determined by ELISA from co-culture supernatants. Wildtype B cells were cultured with or without bone-marrow-derived monocytes/macrophages from wildtype or Stab1-/- mice as indicated. (A-C,E,F) Each data point represents one mouse (A-C) or one cell (E,F) and bars represent the means \pm s.e.m., $n=10$ (wildtype and Stab1 ${ }^{-/-}$) (A, B), 4 (wildtype and Stab $1^{-/-}$) (C), 118 (wildtype) and 140 $\left(S t a b 1^{-/-}\right) \mathbf{( E )}$ and 4 per condition $\mathbf{( F )}$. Statistical significance was determined with Student's unpaired $(\mathbf{A}-\mathbf{C}, \mathbf{E})$ or paired $(\mathbf{F})$ two-tailed $t$-test with Welch's correction when applicable (A), ${ }^{*} P<0.05,{ }^{* \star} P<0.01,{ }^{\star \star *} P<0.001,{ }^{\star \star * \star} P<0.0001$.

imply that Clever-1 in monocytes/macrophages controls the overall activity of $\mathrm{B}$ cells via the production of inflammatory cytokines.
Stab1 $1^{-/-}$mice are born at an expected Mendelian ratio and have no detectable macroscopic or microscopic defects or behavioral abnormalities $(27,30)$. Under physiological 
conditions, only a simultaneous deletion of Stab1 together with its close homolog Stab2 results in glomelurofibrotic nephropathy possibly by impairing the clearance of profibrotic cytokine GDF-15 in the liver (30). Stab1 $1^{-/-}$single-knockout mice show aggravated fibrosis and delayed resolution in liver injury models likely due to impaired myeloid-cell dependent removal of fibrogenic products of lipid peroxidation (25). These mice have also been shown to manifest with diminished tumor progression in melanoma, lymphoma or breast cancer models in vivo, probably due to altered migration of leukocytes to tumors and altered scavenging of certain extracellular matrix molecules $(27,31)$. Stab1 $1^{-/}$mice do not manifest with opportunistic infections, and although they have not been subjected to infection models, short-term and long-term treatments of wildtype mice with function-blocking anti-Clever-1 antibodies did not result in increased rates of spontaneous infections or impairment in the clearance of experimental Staphylococcus aureus skin infections, arguing against an overall increase in the infection susceptibility in the absence of Clever-1 $(12,27)$.

Our current findings thus reveal for the first time the aberrant control of humoral immune responses in Stab1-/mice. Based on the well-known scavenging function of Clever1 , we first hypothesized that in the wildtype setting, antigen scavenging by Clever-1 might limit antigen delivery to $\mathrm{B}$ cells and consequently restrain humoral immune responses. Surprisingly, however, we found that in the spleen, Clever1 was completely absent from all splenic macrophages, including red pulp, MZ and metallophilic macrophages, and was solely expressed by the vascular endothelium. The possible scavenging function of endothelial Clever-1 in the spleen and that of endothelial and macrophagic Clever-1 in other organs is highly unlikely, since the intravenously injected fluorescent TI-2 antigen NP-Ficoll accumulated similarly into MZ macrophages in both wildtype and Stab1-/- mice. Moreover, splenectomy did not abrogate the constitutively higher IgM and IgG levels or the enhanced production of NP-Ficoll-specific IgM post-immunization in Stab1-/- mice. Therefore, our data suggest that the exaggerated humoral immune responses in $S t a b 1^{-/-}$mice are largely of extrasplenic origins.

The enhanced humoral immune responses in Stab1-/mice are likely dependent on the altered functionality of monocytes/macrophages. This notion is supported by the finding that the increased baseline level of IgG was present also in mice in which Clever-1 is selectively deleted from these myeloid cell populations (conditional Stab1 knockout mice expressing Cre recombinase under the $L y z 2$ promoter). We showed here also that the loss of Clever-1 in monocytes/macrophages enhances TNF- $\alpha$ production, and we have previously reported that in humans, Clever-1 $1^{\text {high }}$ monocytes downregulate IFN $\gamma$ and TNF- $\alpha$ production by antigen-specific T cells (16). Neutralizing TNF- $\alpha$ largely reduced the increased capacity of Stab1-/monocytes to support IgM secretion in co-culture experiments. Moreover, Stab1-/- mice manifested with a massive increase in Ifi202b transcription, an interferon-inducible gene (32). These data suggest that Clever-1 serves as an endogenous immunosuppressive molecule in monocytes/macrophages, where it maintains the production of inflammatory cytokines at physiological levels. When Clever-1 is removed from monocytes/macrophages, one consequence of the unrestrained production of inflammatory cytokines is the increased IgM and IgG production by $\mathrm{B}$ cells, possibly as a feedback regulatory response for compensating the actions of Clever-1.

Deleting or neutralizing Clever-1 augments antitumor immune responses at least in melanoma, lymphoma and breast cancer $(27,31)$. The efficacy of anti-Clever-1 antibodies in these experimental models has led to the development of fully human anti-Clever-1 antibodies that are entering clinical trials for cancer immunotherapy. Thus, our current observations suggest that disrupting the function of Clever-1 should not lead to the suppression of antibody production, and, in fact, could even improve humoral immune responses against tumor antigens.

\section{ETHICS STATEMENT}

All animal experiments were approved by the local Committee for Animal Experimentation in Finland (license numbers 5587/04.10.07/2014 and 5762/04.10.07/2017) and were performed according to the $3 \mathrm{Rs}$ principles and in accordance with the Finnish Act on Animal Experimentation $(62 / 2006)$.

\section{AUTHOR CONTRIBUTIONS}

JD, SJ, and MS conceived the study project and designed experiments. JD, MV, MK, PR, RV, KE, and $\mathrm{MH}$ performed experiments and analyzed the data. JD, MV, and MS drafted the manuscript. All authors contributed to the interpretation of the experiments, critically reviewed the manuscript, and gave final approval of the work.

\section{FUNDING}

This study was supported by research grants from the Academy of Finland (to SJ and MS).

\section{ACKNOWLEDGMENTS}

We thank Sari Mäki, Mari Parsama, and Teija Kanasuo for excellent technical assistance and Dr. Kalle-Pekka Nera for fruitful discussions.

\section{SUPPLEMENTARY MATERIAL}

The Supplementary Material for this article can be found online at: https://www.frontiersin.org/articles/10.3389/fimmu. 2018.02257/full\#supplementary-material 


\section{REFERENCES}

1. Kzhyshkowska J, Gratchev A, Goerdt S. Stabilin-1, a homeostatic scavenger receptor with multiple functions. J Cell Mol Med. (2006) 10:635-49. doi: $10.1111 /$ j.1582-4934.2006.tb00425.x

2. Goerdt S, Walsh LJ, Murphy GF, Pober JS. Identification of a novel high molecular weight protein preferentially expressed by sinusoidal endothelial cells in normal human tissues. J Cell Biol. (1991) 113:1425-37. doi: 10.1083/jcb.113. 6.1425

3. Goerdt S, Bhardwaj R, Sorg C. Inducible expression of MS-1 high-molecularweight protein by endothelial cells of continuous origin and by dendritic cells/macrophages in vivo and in vitro. Am J Pathol. (1993) 142:1409-22.

4. Adachi H, Tsujimoto M. FEEL-1, a novel scavenger receptor with in vitro bacteria-binding and angiogenesis-modulating activities. J Biol Chem. (2002) 277:34264-70. doi: 10.1074/jbc.M204277200

5. Irjala H, Elima K, Johansson EL, Merinen M, Kontula K, Alanen K, et al. The same endothelial receptor controls lymphocyte traffic both in vascular and lymphatic vessels. Eur J Immunol. (2003) 33:815-24. doi: $10.1002 /$ eji.200323859

6. Kzhyshkowska J, Gratchev A, Brundiers H, Mamidi S, Krusell L, Goerdt S. Phosphatidylinositide 3-kinase activity is required for stabilin-1mediated endosomal transport of acLDL. Immunobiology (2005) 210:161-73. doi: 10.1016/j.imbio.2005.05.022

7. Kzhyshkowska J, Workman G, Cardo-Vila M, Arap W, Pasqualini R, Gratchev A, et al. Novel function of alternatively activated macrophages: stabilin-1-mediated clearance of SPARC. J Immunol. (2006) 176:5825-32. doi: 10.4049/jimmunol.176.10.5825

8. Kzhyshkowska J, Gratchev A, Schmuttermaier C, Brundiers H, Krusell L, Mamidi S, et al. Alternatively activated macrophages regulate extracellular levels of the hormone placental lactogen via receptormediated uptake and transcytosis. J Immunol. (2008) 180:3028-37. doi: 10.4049 /jimmunol.180.5.3028

9. Mosig S, Rennert K, Krause S, Kzhyshkowska J, Neunubel K, Heller R, et al. Different functions of monocyte subsets in familial hypercholesterolemia: potential function of $\mathrm{CD} 14+\mathrm{CD} 16+$ monocytes in detoxification of oxidized LDL. Faseb J. (2009) 23:866-74. doi: 10.1096/fj.08118240

10. Kzhyshkowska J. Multifunctional receptor stabilin-1 in homeostasis and disease. ScientificWorldJournal (2010) 10:2039-53. doi: 10.1100/tsw.2010.189

11. Salmi M, Koskinen K, Henttinen T, Elima K, Jalkanen S. CLEVER-1 mediates lymphocyte transmigration through vascular and lymphatic endothelium. Blood (2004) 104:3849-57. doi: 10.1182/blood-2004-01-0222

12. Karikoski M, Irjala H, Maksimow M, Miiluniemi M, Granfors K, Hernesniemi S, et al. Clever-1/Stabilin-1 regulates lymphocyte migration within lymphatics and leukocyte entrance to sites of inflammation. Eur J Immunol. (2009) 39:3477-87. doi: 10.1002/eji.200939896

13. Shetty S, Weston CJ, Oo YH, Westerlund N, Stamataki Z, Youster $\mathrm{J}$, et al. Common lymphatic endothelial and vascular endothelial receptor-1 mediates the transmigration of regulatory $\mathrm{T}$ cells across human hepatic sinusoidal endothelium. J Immunol. (2011) 186:4147-55. doi: 10.4049/jimmunol.1002961

14. Shetty S, Bruns T, Weston CJ, Stamataki Z, Oo YH, Long HM, et al. Recruitment mechanisms of primary and malignant $B$ cells to the human liver. Hepatology (2012) 56:1521-31. doi: 10.1002/hep.25790

15. Palani S, Maksimow M, Miiluniemi M, Auvinen K, Jalkanen S, Salmi M. Stabilin-1/CLEVER-1, a type 2 macrophage marker, is an adhesion and scavenging molecule on human placental macrophages. Eur J Immunol. (2011) 41:2052-63. doi: 10.1002/eji.201041376

16. Palani S, Elima K, Ekholm E, Jalkanen S, Salmi M. Monocyte stabilin-1 suppresses the activation of Th1 lymphocytes. J Immunol. (2016) 196:115-23. doi: 10.4049/jimmunol.1500257

17. Coutinho A, Kazatchkine MD, Avrameas S. Natural autoantibodies. Curr Opin Immunol. (1995) 7:812-8. doi: 10.1016/0952-7915(95)80053-0

18. Baumgarth $\mathrm{N}$. The double life of a B-1 cell: self-reactivity selects for protective effector functions. Nat Rev Immunol. (2011) 11:34-46. doi: 10.1038/ nri2901
19. Koppel EA, Litjens M, van den Berg VC, van Kooyk Y, Geijtenbeek TB. Interaction of SIGNR1 expressed by marginal zone macrophages with marginal zone $B$ cells is essential to early $\operatorname{IgM}$ responses against Streptococcus pneumoniae. Mol Immunol. (2008) 45:2881-7. doi: 10.1016/j.molimm.2008.01.032

20. Ehrenstein MR, Notley CA. The importance of natural IgM: scavenger, protector and regulator. Nat Rev Immunol. (2010) 10:778-86. doi: $10.1038 /$ nri2849

21. Cerutti A, Cols M, Puga I. Marginal zone B cells: virtues of innatelike antibody-producing lymphocytes. Nat Rev Immunol. (2013) 13:118-32. doi: $10.1038 /$ nri3383

22. Nutt SL, Hodgkin PD, Tarlinton DM, Corcoran LM. The generation of antibody-secreting plasma cells. Nat Rev Immunol. (2015) 15:160-71. doi: $10.1038 /$ nri3795

23. Rantakari P, Auvinen K, Jappinen N, Kapraali M, Valtonen J, Karikoski M, et al. The endothelial protein PLVAP in lymphatics controls the entry of lymphocytes and antigens into lymph nodes. Nat Immunol. (2015) 16:386-96. doi: $10.1038 /$ ni.3101

24. Schledzewski K, Falkowski M, Moldenhauer G, Metharom P, Kzhyshkowska J, Ganss R, et al. Lymphatic endothelium-specific hyaluronan receptor LYVE1 is expressed by stabilin-1+, F4/80+, CD11b+ macrophages in malignant tumours and wound healing tissue in vivo and in bone marrow cultures in vitro: implications for the assessment of lymphangiogenesis. J Pathol. (2006) 209:67-77. doi: 10.1002/path.1942

25. Rantakari P, Patten DA, Valtonen J, Karikoski M, Gerke H, Dawes H, et al. Stabilin-1 expression defines a subset of macrophages that mediate tissue homeostasis and prevent fibrosis in chronic liver injury. Proc Natl Acad Sci USA. (2016) 113:9298-303. doi: 10.1073/pnas.1604780113

26. Guinamard R, Okigaki M, Schlessinger J, Ravetch JV. Absence of marginal zone B cells in Pyk-2-deficient mice defines their role in the humoral response. Nat Immunol. (2000) 1:31-6. doi: 10.1038/76882

27. Karikoski M, Marttila-Ichihara F, Elima K, Rantakari P, Hollmen M, et al. Clever-1/stabilin-1 controls cancer growth and metastasis. Clin Cancer Res. (2014) 20:6452-64. doi: 10.1158/1078-0432.CCR-14-1236

28. Jelinek D F, Lipsky PE. Enhancement of human B cell proliferation and differentiation by tumor necrosis factor-alpha and interleukin 1. J Immunol. (1987) 139:2970-6.

29. Kehrl JH, Miller A, Fauci AS. Effect of tumor necrosis factor alpha on mitogen-activated human B cells. J Exp Med. (1987) 166:786-91. doi: $10.1084 /$ jem.166.3.786

30. Schledzewski K, Geraud C, Arnold B, Wang S, Grone HJ, Kempf T, et al. Deficiency of liver sinusoidal scavenger receptors stabilin-1 and-2 in mice causes glomerulofibrotic nephropathy via impaired hepatic clearance of noxious blood factors. J Clin Invest. (2011) 121:703-14. doi: 10.1172/JCI44740

31. Riabov V, Yin S, Song B, Avdic A, Schledzewski K, Ovsiy I, et al. Stabilin1 is expressed in human breast cancer and supports tumor growth in mammary adenocarcinoma mouse model. Oncotarget (2016) 7:31097-110. doi: 10.18632 /oncotarget. 8857

32. Choubey D, Lengyel P. Interferon action: cytoplasmic and nuclear localization of the interferon-inducible $52-\mathrm{kD}$ protein that is encoded by the If 200 gene from the gene 200 cluster. J Interferon Res. (1993) 13:43-52. doi: 10.1089/jir.1993.13.43

Conflict of Interest Statement: MH, SJ, and MS are shareholders in Faron Pharmaceuticals.

The remaining authors declare that the research was conducted in the absence of any commercial or financial relationships that could be construed as a potential conflict of interest.

Copyright (c) 2018 Dunkel, Viitala, Karikoski, Rantakari, Virtakoivu, Elima, Hollmén, Jalkanen and Salmi. This is an open-access article distributed under the terms of the Creative Commons Attribution License (CC BY). The use, distribution or reproduction in other forums is permitted, provided the original author(s) and the copyright owner(s) are credited and that the original publication in this journal is cited, in accordance with accepted academic practice. No use, distribution or reproduction is permitted which does not comply with these terms. 